\title{
Transcriptomic and proteomic analysis of the human kidney provides a reference dataset and demonstrates profound differences between cortex and medulla
}

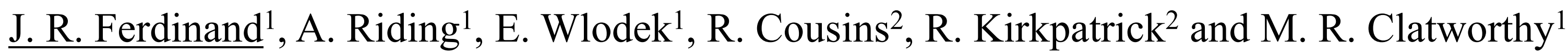 \\ Author affiliations: $\quad 1$ - Molecular Immunity Unit, University of Cambridge, Cambridge, UK \\ 2 - The Pipelines Futures Group, 1250 South Collegeville Road, Collegeville, PA, USA and Gunnels Wood Road, Stevenage, UK
}

Introduction: The use of more marginal donors to combat organ shortage can result in sub-optimal long-term graft function. There is a need to identify biomarkers that predict outcomes, and to understand the mechanisms that lead to good or poor allograft function. Global transcriptomics and proteomics provide hypothesis-free methods to identify biomarkers and pathways. To facilitate their use in kidney transplantation, we first sought to establish a reference dataset to determine inter-individual variation, the relationship between the transcriptome and proteome, and the extent to which variation in the anatomical region of the kidney sampled (cortex versus medulla) might impact the data generated.

Methods: Paired cortex and medulla samples were isolated from $\mathrm{N}=5$ human kidneys donated for transplantation, but deemed unsuitable for use. RNASeq was used to assess gene transcription and the proteome analysed by triple mass spectroscopy.

Results: Principle component analysis of the transcriptomic data showed that the biggest contributor to variance was the anatomical site from which the sample was obtained (ie, cortex vs medulla). Of the 22,655 genes identified, and after correction for multiple testing, 2913 genes were found to be significantly (adjusted $\mathrm{p}$ value (padj) $<0.05$ ) enriched in the cortex and 2282 in the medulla (Figure 1).
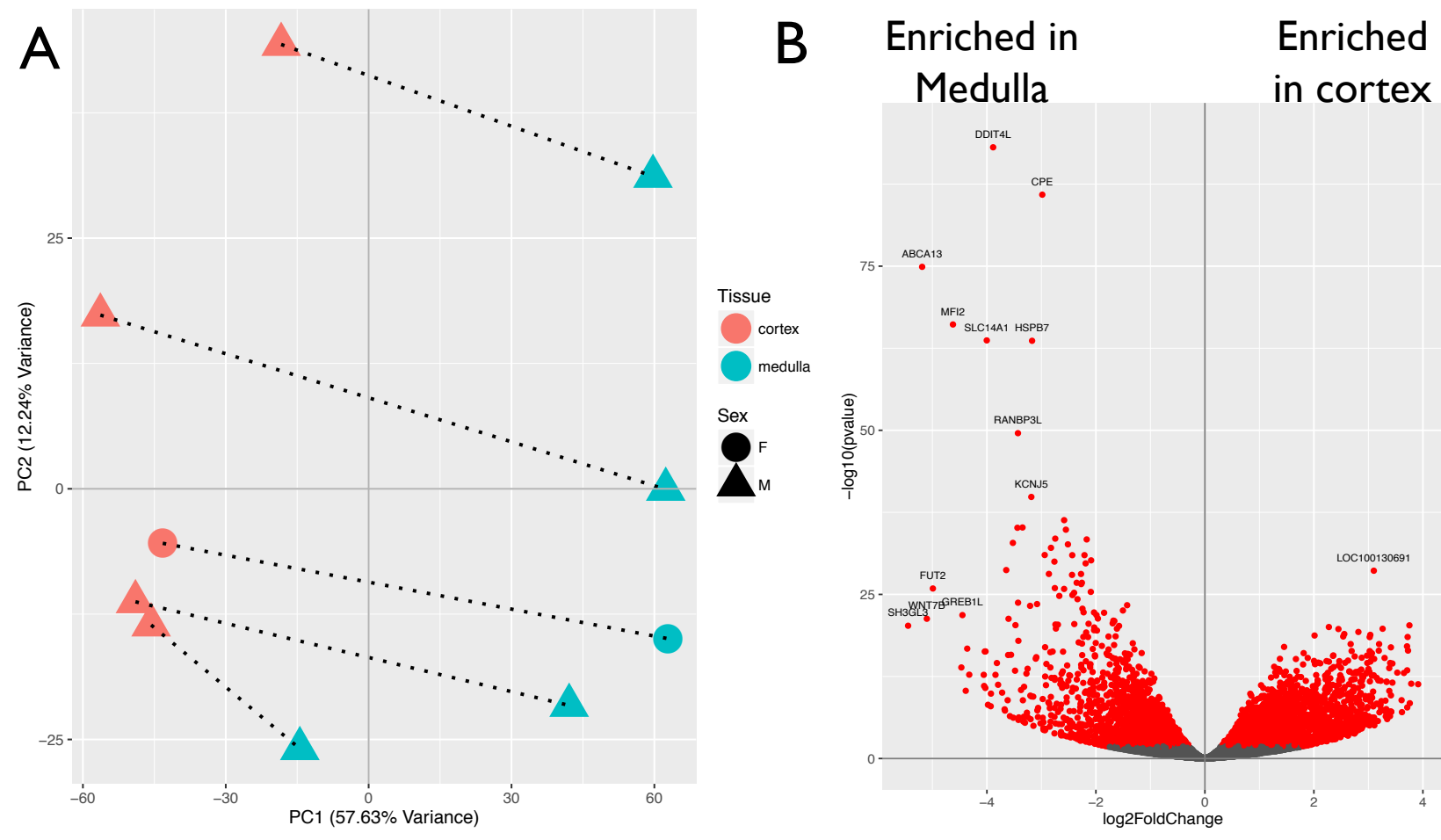

Figure 1 - RNASeq analysis of human kidney. RNA was extracted from tissue taken from the cortex and RNASeq analysis performed. $\boldsymbol{A}$ PCA analysis. Dashed lines indicate paired samples $\boldsymbol{B}$ Volcano plot of data. Significantly enriched genes shown in red.

Gene set enrichment analysis revealed an enrichment of pathways associated with metabolism and NF-kB signalling in the cortex and KRas signalling in the medulla (Figure 2).

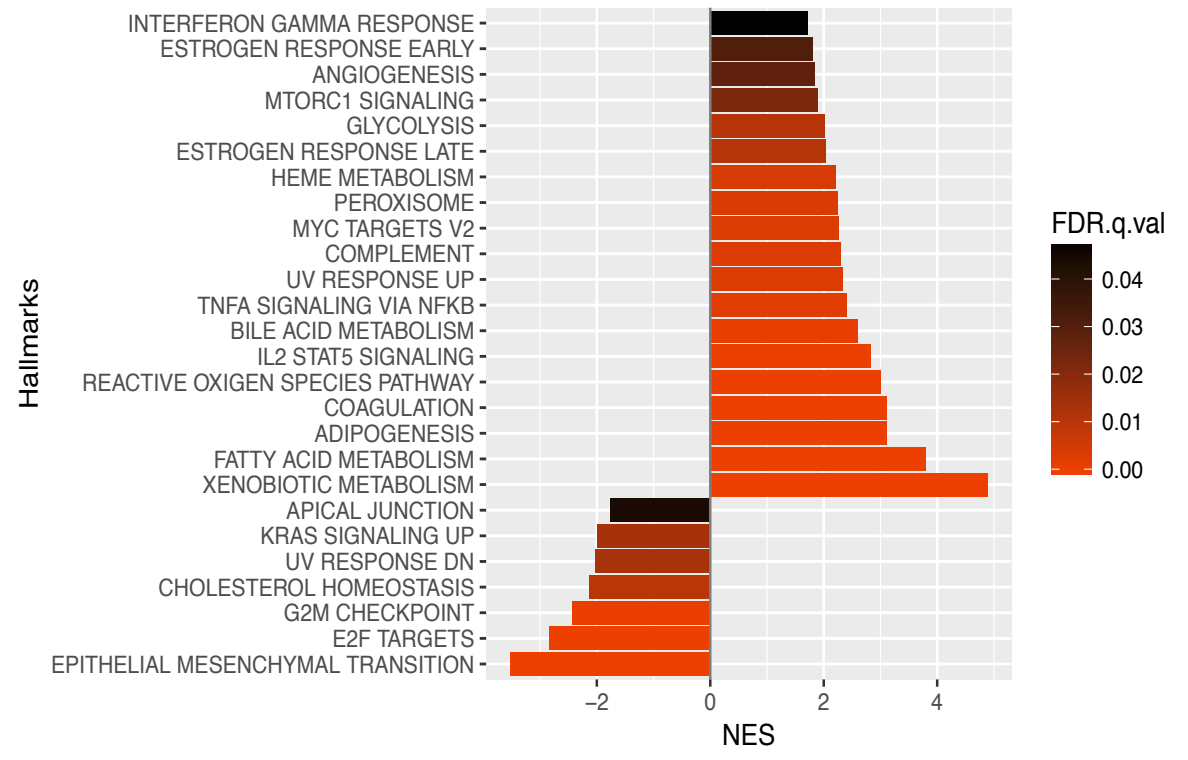

Figure 2-Gene set enrichment analysis. Genes were ranked relative to the medulla and compared to the pathways in the hallmarks database (MSigDB, Broad)

To assess the relationship between the proteome and the transcriptome we performed mass spectroscopy. We were able to confidently identify 4288 proteins (Figure 3). We found that the relative expression between the cortex and the medulla was conserved between the samples(Figure 4). Further we found that many pathways identified as enriched in either tissue by Gene set enrichment analysis were conserved (Data not shown)
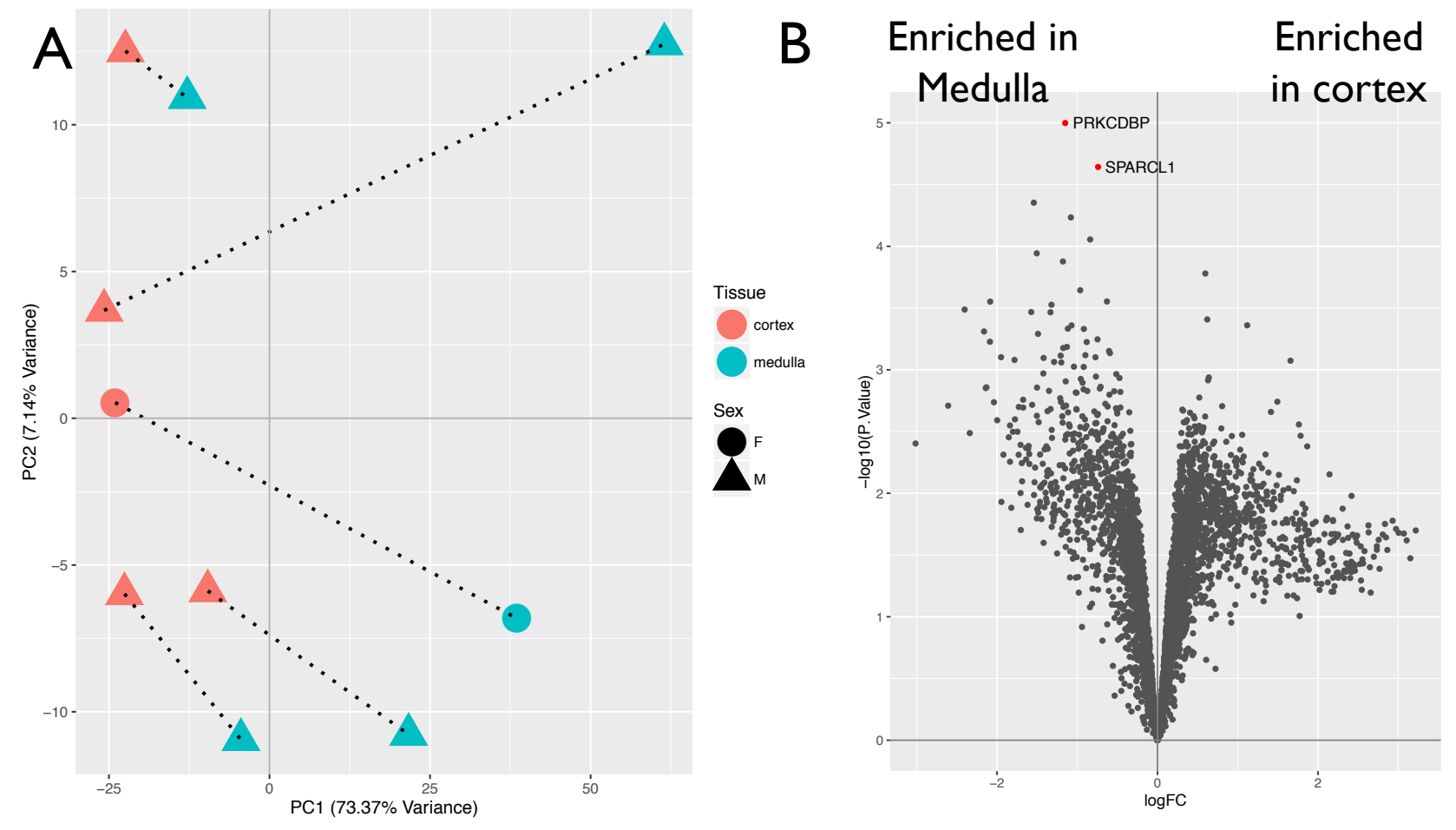

Figure 3 - Mass Spec analysis of human kidney. A PCA analysis. Dashed lines indicate paired samples $\boldsymbol{B}$ Volcano plot of data. Significantly enriched genes shown in red.

A

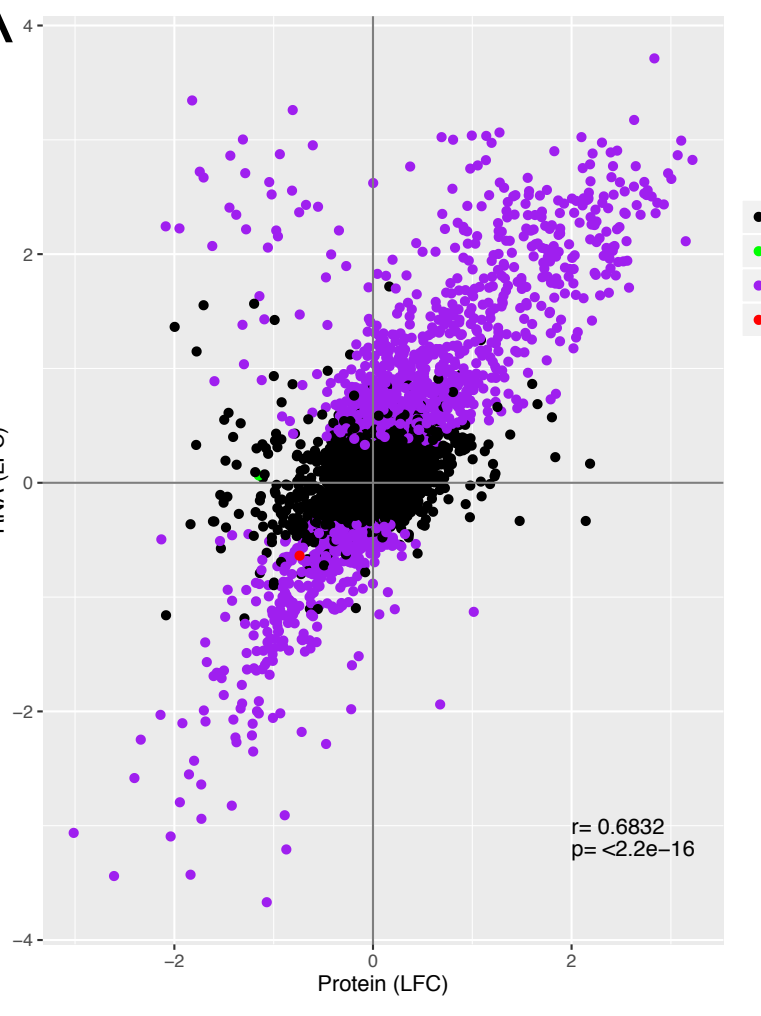

$\mathrm{B}$

RNA
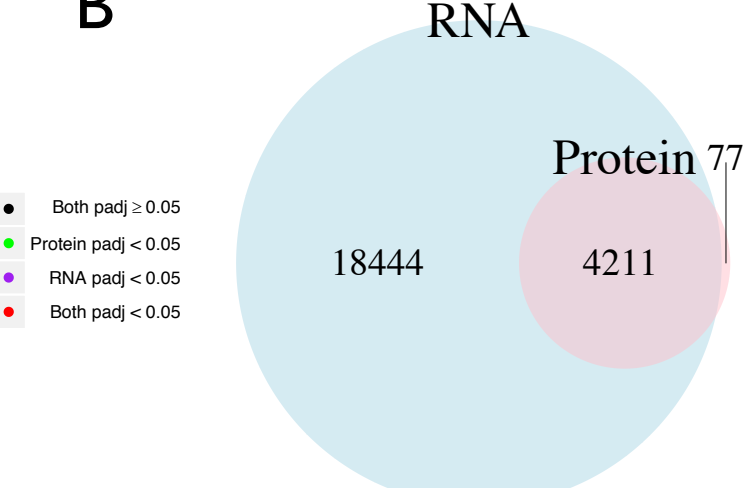

Figure 4 - Transcriptome

Proteome comparison. A- $\log$ fold change in expression of the transcriptome and proteome relative to Medulla. B Numbers of genes and proteins identified

To identify 'medullary contamination' of needle core biopsies we propose the genes highlighted below could be used either at the whole transcriptome level (Figure 5) or via qPCR(Figure 5B * genes).
A
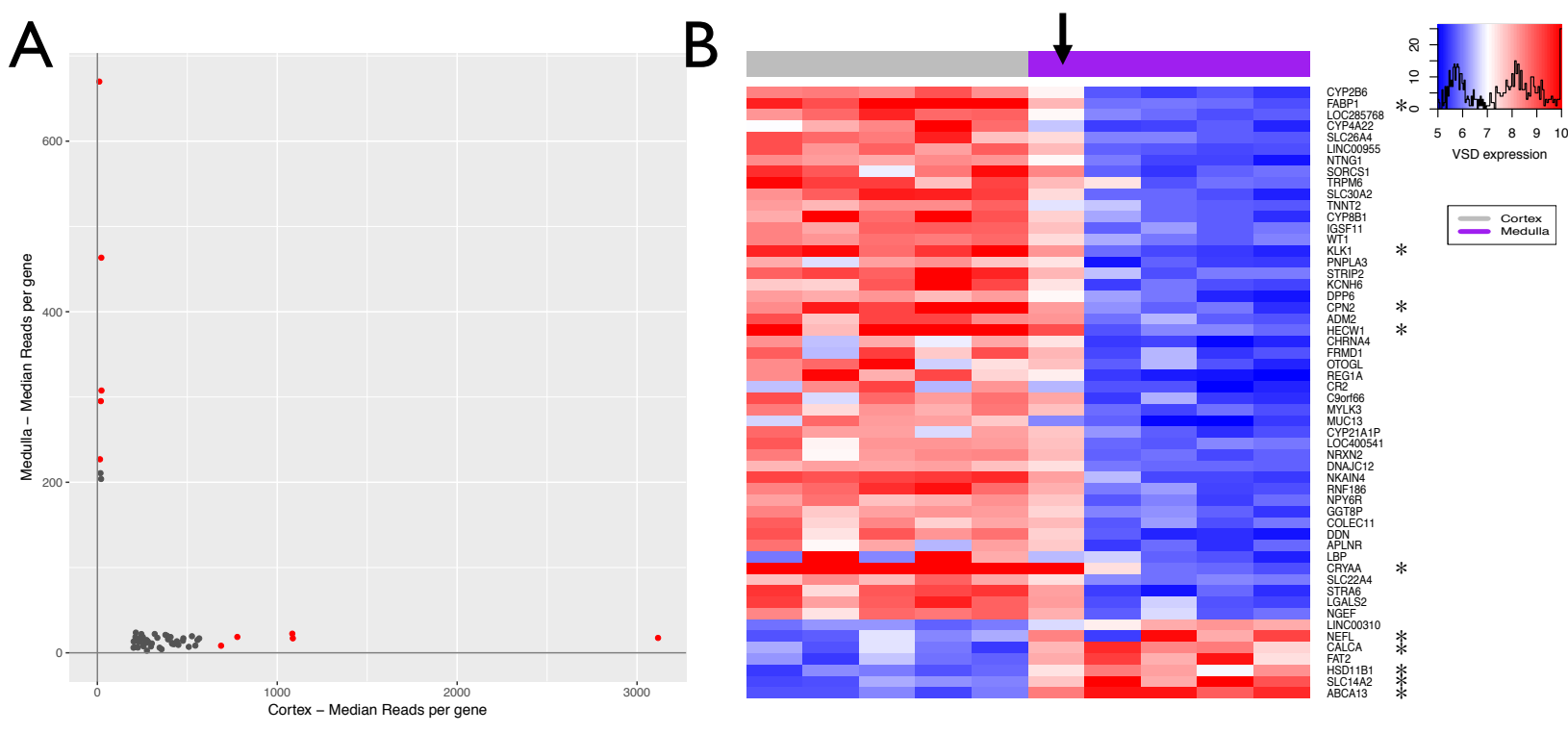

Figure 5 - Genes to identify medullary contamination. Genes were filtered for padj < 0.05, Median expression for Medulla $>200$ and cortex $<25$ or the reverse $(\boldsymbol{A})$. Candidates were plotted as a heat map $(\boldsymbol{B})$. Sample marked by an arrow indicates contamination. ${ }^{*}$ or red points are candidates for a qPCR based screening approach.

Discussion: These results have a number of important implications; since the variation in gene expression between individuals is smaller than the variation within the tissue, differences in the amount of medullary tissue in a core biopsy will change the gene signature independent of any intervention or pathway activated. Our dataset allows the generation of a gene list that can be used to quantify 'medullary contamination'. Furthermore, the close alignment of transcript \& protein levels suggests that transcriptomic studies (which are less costly, and have a more global coverage) provide a useful method to identify genes that could subsequently be taken forward as protein biomarkers. 